\title{
Isolation and characterization of $\alpha$-enolase, a novel fibronectin-binding protein from Streptococcus suis
}

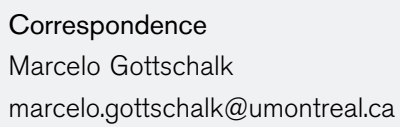

Received 28 January 2008

Revised 5 May 2008

Accepted 29 May 2008

\author{
Miriam Esgleas, ${ }^{1}$ Yuanyi Li, ${ }^{1}$ Mark A. Hancock, ${ }^{2}$ Josée Harel, ${ }^{1}$ \\ J. Daniel Dubreuil ${ }^{1}$ and Marcelo Gottschalk ${ }^{1}$
}

\begin{abstract}
${ }^{1}$ Groupe de Recherche sur les Maladies Infectieuses du Porc (GREMIP) and Centre de Recherche en Infectiologie Porcine (CRIP), Faculté de Médecine Vétérinaire, Université de Montréal, Québec, Canada

${ }^{2}$ Sheldon Biotechnology Centre, McGill University, Montreal, Québec, Canada
\end{abstract}

\begin{abstract}
Streptococcus suis is an important swine pathogen that causes meningitis, endocarditis, arthritis and septicaemia. As a zoonotic agent, $S$. suis also causes similar diseases in humans. Binding of pathogenic bacteria to extracellular matrix components enhances their adhesion to and invasion of host cells. In the present study we isolated and identified a novel fibronectin-binding protein from S. suis. The native protein (designated SsEno) possessed not only high homology with other bacterial enolases but also enolase activity. We cloned, expressed and purified SsEno and showed that it is ubiquitously expressed by all $S$. suis serotypes and we identified its surface localization using immunoelectron microscopy. ELISA demonstrated that SsEno binds specifically to fibronectin and plasminogen in a lysine-dependent manner. Additional surface plasmon resonance assays demonstrated that SsEno binds to fibronectin or plasminogen with low nanomolar affinity. Inhibition experiments with anti-SsEno antibodies also showed that bacterial SsEno is important for the adhesion to and invasion of brain microvascular endothelial cells by S. suis. Overall, the present work is the first study, to our knowledge, to demonstrate a fibronectinbinding activity of a bacterial enolase, and shows that, similar to other bacterial fibronectin-binding proteins, SsEno may contribute to the virulence of $S$. suis.
\end{abstract}

\section{INTRODUCTION}

Streptococcus suis is a major swine pathogen that causes septicaemia, meningitis, endocarditis and arthritis (Higgins \& Gottschalk, 2005). Of the 35 known serotypes, serotype 2 is the most frequently isolated and associated with disease (Higgins \& Gottschalk, 2005). It has been proposed that two serotypes (serotypes 32 and 34 ) be excluded from $S$. suis and redesignated Streptococcus orisratti (Hill et al., 2005). S. suis, especially serotype 2 , has also been described as an important zoonotic agent that affects people in close contact with infected pigs or pork-derived products (Lun et al., 2007). Indeed, an important number of cases of human disease with a high rate of mortality in China were linked directly to a concurrent outbreak of $S$. suis infection in pigs (Ye et al., 2006).

Little is known about $S$. suis virulence factors. The capsule polysaccharide (CPS) is a critical virulence factor, given that unencapsulated isogenic mutants are completely

Abbreviations: EACA, $\varepsilon$-amino- $n$-caproic acid; ECM, extracellular matrix; FBPS, fibronectin-fibrinogen-binding protein; GAPDH, glyceraldehyde3-phosphate dehydrogenase; PBMEC, porcine brain microvascular endothelial cell; RU, relative units; SEN, Streptococcus pyogenes enolase; SPR, surface plasmon resonance. avirulent and rapidly cleared from the circulation in pig and mouse infection models (Charland et al., 2000; Smith et al., 1999). However, non-virulent strains are also encapsulated, indicating that the virulence of this pathogen is a multifactorial process (Gottschalk \& Segura, 2000). Other potential virulence factors have also been described in S. suis, including a haemolysin (suilysin), a $136 \mathrm{kDa}$ muramidase-released protein (MRP), a $110 \mathrm{kDa}$ extracellular factor (EF) protein, a hyaluronidase, a superoxide dismutase, various proteases, a serum opacity factor and different adhesins (Baums et al., 2006; Segura \& Gottschalk, 2004).

The pathogenesis of $S$. suis infection is not fully understood and likely involves many steps (Gottschalk \& Segura, 2000). Binding between bacterial adhesins and host proteins is an essential step in the colonization of mucosal surfaces, and has also been implicated in bacterial invasion of host cells (Tamura et al., 1994). Indeed, S. suis is able to bind to, and in some cases invade, endothelial and epithelial cells of human and porcine origin (Benga et al., 2005; Gottschalk \& Segura, 2000; Lalonde et al., 2000; Norton et al., 1999; Vanier et al., 2004). However, the specific mechanisms involved in these interactions are unknown. 
Some pathogens use host extracellular matrix (ECM) proteins to potentiate their virulence. The ECM is a stable macromolecular structure underlying epithelial and endothelial cells and surrounding connective tissue cells (Westerlund \& Korhonen, 1993). Its composition varies among different organs, but the main components are fibronectin, collagen, elastin, laminin and glycosaminoglycans (Kreis \& Vale, 1993). Many of these proteins can potentially serve as surface receptors for bacterial binding to host cells via their adhesins (Joh et al., 1999; SchwarzLinek et al., 2004; Westerlund \& Korhonen, 1993). S. suis is able to adhere to fibronectin and different types of collagens (Esgleas et al., 2005). In fact, a $64 \mathrm{kDa}$ fibronectin-fibrinogen-binding protein (FBPS) with binding capacity for these two host proteins has been described for S. suis (de Greeff et al., 2002). The role of FBPS in bacterial pathogenesis is not well understood. However, studies with the $f b p s$ mutant suggest that this protein could play a role in $S$. suis colonization of various organs (de Greeff et al., 2002).

Another S. suis adhesin that is able to bind to different host proteins such as plasminogen and albumin is the $39 \mathrm{kDa}$ glyceraldehyde-3-phosphate dehydrogenase (GAPDH) (Jobin et al., 2004; Quessy et al., 1997). Evidence that this protein is involved in the binding of $S$. suis to host tissues has been provided by the finding that mutants deficient in the surface expression of GAPDH have decreased binding to tracheal cells and porcine tracheal rings (Brassard et al., 2001, 2004). GAPDH is also a cytosolic enzyme that participates in the degradation of carbohydrates via glycolysis and other catabolic pathways, as well as in glucose synthesis via gluconeogenesis. Another enzyme essential for glycolysis and gluconeogenesis is enolase (Marks, 1998). This cytosolic enzyme catalyses the conversion of 2-phosphoglycerate (2-PG) into phosphoenolpyruvate (PEP) via the elimination of a water molecule (Wold \& Ballou, 1957). Enolase has also been identified as a novel plasminogen receptor on the surface of many eukaryotic cells (Miles et al., 1991; Nakajima et al., 1994). This unexpected feature has also been described in several bacteria (such as Staphylococcus aureus and many streptococci), fungi and nematodes (Bergmann et al., 2001; Jolodar et al., 2003; Jong et al., 2003; Molkanen et al., 2002; Pancholi \& Fischetti, 1998; Sharma et al., 2006). While the role of plasminogen as an important component in streptoccoci adhesion is well established (Pancholi et al., 2003), the role of enolase in bacterial adherence and host cell invasion has not, to our knowledge, been investigated previously.

In addition to identifying and inducing the expression of $S$. suis $\alpha$-enolase (SsEno), we now demonstrate for the first time, to our knowledge, that this protein not only has plasminogen-binding activity but also has important fibronectin-binding properties. Finally, we also demonstrate that surface SsEno, which is present in all the described serotypes of $S$. suis, may play an important role in the processes of adhesion and invasion of host endothelial cells.

\section{METHODS}

Bacterial strains and growth conditions. Reference strains from the 35 different $S$. suis serotypes came from our collection. Highly virulent strain $166^{\prime}$ of $S$. suis serotype 2 (Berthelot-Herault et al., 2005) was kindly provided by Dr M. Kobisch (AFSSA, Ploufragan, France). S. suis strains were grown in Todd-Hewitt broth (THB) (BD) as described previously (Al-Numani et al., 2003). Lateexponential-phase bacteria were washed in PBS, pH 7.3. Escherichia coli strain BL21DE3 was used for expression experiments. E. coli was grown in Luria-Bertani broth or on agar plates. Ampicillin $(100 \mu \mathrm{g}$ $\mathrm{ml}^{-1}$; Roche) was used in growth media when required.

\section{Isolation of $\mathbf{5 2} \mathbf{~ k D a}$ protein by fibronectin-affinity chromato-} graphy. Strain $166^{\prime}$ was harvested from THB by centrifugation at $5500 \boldsymbol{g}$ for $15 \mathrm{~min}$, washed twice in PBS $(0.01 \mathrm{M}, \mathrm{pH} 7.4)$, and resuspended in HEPES buffer $(10 \mathrm{mM}, \mathrm{pH} 7.4)$. Bacteria were disrupted by sonicating the bacterial suspension with an ultrasonic probe (Sonics \& Materials) on ice for 30 min with $15 \mathrm{~s}$ intervals of cooling on ice at $80 \%$ duty cycle. Cell debris and non-lysed bacteria were removed by centrifugation. Supernatant proteins were concentrated to a volume of $<1.0 \mathrm{ml}$ using Amicon Ultra-15 concentrators (Millipore) and applied to a $5 \mathrm{ml}$ fibronectin-coupled $\mathrm{CNBr}$ activated Sepharose 4B column (Amersham Biosciences) preequilibrated with $0.1 \mathrm{M}$ Tris/ $\mathrm{HCl}$ buffer, $\mathrm{pH}$ 8.0. After washing with five column-volumes of this buffer, bound proteins were eluted with $30 \mathrm{ml} 8 \mathrm{M}$ urea. Starting lysates and fractionated samples were analysed by SDS-PAGE ( $12.5 \%$ polyacrylamide) under reducing conditions, followed by Coomassie blue staining. Protein concentrations were determined by the Lowry method (Lowry et al., 1951). Samples were maintained at $-70{ }^{\circ} \mathrm{C}$ until analysis.

$\mathrm{N}$-terminal sequencing was carried out with chromatographic fractions containing fibronectin-binding proteins after being electroblotted onto PVDF membranes (Immobilon-P; Millipore) and visualized by staining with $0.1 \%$ Ponceau S (Sigma-Aldrich) in $1 \%$ acetic acid. Bands of interest were excised, destained with distilled water, and subjected to peptide sequencing at the Biotechnology Research Institute (Montreal, Canada).

Cloning, expression and purification of recombinant SsEno (rSsEno). The peptide sequence identified by $\mathrm{N}$-terminal sequencing of the predominant fibronectin-binding protein was subjected to a homology search using the BLAST program against the strain $\mathrm{P}$ 1/7 genome sequence of S. suis (http://www.sanger.ac.uk/Projects/S_suis/). A database search revealed $100 \%$ homology to the $\mathrm{N}$-terminal region of a $1308 \mathrm{bp}$ ORF that corresponds to the S. suis enolase (SsEno). The sequence was used to design primers for PCR amplification of the SsEno gene. The primers used were the Eno1forward primer 5'-TATAAGGATCCTTGTCAATTATTACTGATGTTTACGC-3', introducing a BamHI site (bold and underlined letters), and the Eno2-reverse primer 5'-TATAAAGCTTTTATTTTTTCAAGTTGTAGAATGAGTTCAAGCC-3', introducing a HindIII site (bold and underlined letters). Automated sequencing was then used to check the amplified enolase gene. The verified complete gene was cloned into pET-32a (Novagen) using the BamHI and HindIII sites. This plasmid contains a His-tag-encoding sequence of about $25 \mathrm{kDa}$. The plasmid pET-32a-SsEno was introduced into E. coli Bl21DE3 for IPTG-inducible expression of recombinant SsEno. Under native conditions, the His-tagged fusion protein was purified by HisTrap chromatography according to the manufacturer's protocols (Amersham Biosciences). Protein-containing fractions were determined by SDS-PAGE ( $12.5 \%$ polyacrylamide) followed by Coomasie blue staining. Purified rSsEno was used to produce polyclonal antibodies to SsEno in rabbits, as described previously (Li et al., 2006). To prepare affinity-purified anti-SsEno IgG, the polyclonal 
serum was subjected to purification on protein-A Sepharose CL-4B (Amersham Pharmacia) (Pancholi \& Fischetti, 1998).

Enolase activity. $\alpha$-Enolase activity was determined by measuring the transformation of $\mathrm{NADH} \cdot \mathrm{H}^{+}$to $\mathrm{NAD}^{+}$, as described elsewhere (Pancholi \& Fischetti, 1998) with some modifications. Briefly, the enzymic reactions were performed at $37{ }^{\circ} \mathrm{C}$ in $100 \mathrm{mM}$ HEPES buffer, $\mathrm{pH} 7.0$, containing $5.0 \mathrm{mM} \mathrm{MgSO}, 0.2 \mathrm{mM} \mathrm{NADH}$ (Roche), $0.25 \mathrm{mM}$ 2-phosphoglycerate (2-PGE) (Sigma), $1.2 \mathrm{mM}$ ADP (Roche), 10.7 IU lactate dehydrogenase (Roche) and 2.5 IU pyruvate kinase (Roche), in a final volume of $1 \mathrm{ml}$. The reaction was started by adding $100 \mu \mathrm{l}$ of test solution containing rSsEno $(0-20 \mu \mathrm{g})$. The $\alpha$ enolase activity was measured in terms of the rate of reduction in the $A_{340}$ (i.e. increase in the production of $\mathrm{NAD}$ from $\mathrm{NADH} \cdot \mathrm{H}^{+}$). Rabbit muscle enolase (Sigma) was used as a positive control and buffer without enolase was used as a negative control.

Localization of SsEno in S. suis. To evaluate the presence of SsEno in different bacterial compartments, S. suis strain 166' was grown overnight at $37^{\circ} \mathrm{C}$ with agitation in $30 \mathrm{ml}$ THB. The subcellular components (supernatant, cell wall, cytoplasmic and membrane fractions) were fractionated with mutanolysin by using the method of Yother \& White (1994). Equivalent amounts of all fractions were analysed by SDS-PAGE ( $12.5 \%$ polyacrylamide) and Western blotting using optimally diluted rabbit anti-SsEno IgG.

The surface exposure of SsEno was examined by using immunelectron microscopy. Briefly, S. suis $166^{\prime}$ was grown in $5 \mathrm{ml}$ THB until late exponential phase, centrifuged, and resuspended in PBS ( $\mathrm{pH}$ 8.0). A $20 \mu \mathrm{l}$ aliquot of the bacterial suspension was placed on nickelFormvar grids (INRS, Institut Armand Frappier, Laval, Canada) and allowed to partially air dry. After blocking for 30 min with $10 \%$ normal donkey serum in dilution buffer (PBS, pH 8.0, $1 \%$ BSA, $1 \%$ Tween 20), the grids were incubated with optimally diluted rabbit anti-SsEno IgG for $2 \mathrm{~h}$ at room temperature. The grids were washed three times with PBS, pH 8.0, $1 \%$ Tween 20 and incubated for $1 \mathrm{~h}$ at room temperature with $12 \mathrm{~nm}$ colloidal gold-Affinipure donkey antirabbit IgG (Jackson ImmunoResearch Laboratories). After several washes with PBS, $1 \%$ Tween 20 , bacteria were stained with $1 \%$ phosphotungstic acid and examined with an electron microscope (Philips 201) at an accelerating voltage of $60 \mathrm{kV}$. Bacteria coated with normal rabbit antibodies served as a negative control.

rSsEno binding assays for plasminogen and fibronectin. Purified rSsEno binding to immobilized fibronectin (Roche) and plasminogen (Sigma) was first determined by ELISA. Maxisorp flatbottomed microtitre 96-well plates (Nunc) were coated with $100 \mu \mathrm{l}$ of serial dilutions of fibronectin or plasminogen, depending on the experiment, in $0.1 \mathrm{M}$ carbonate coating buffer $[0.15 \%(\mathrm{w} / \mathrm{v})$ $\mathrm{Na}_{2} \mathrm{CO}_{3}, \quad 0.1 \% \quad(\mathrm{w} / \mathrm{v}) \quad \mathrm{MgCl}_{2} \cdot 6 \mathrm{H}_{2} \mathrm{O}, \quad 0.3 \% \quad(\mathrm{w} / \mathrm{v}) \quad \mathrm{NaHCO}_{3}$ $\left(\mathrm{pH}\right.$ 9.6)] and incubated overnight at $4{ }^{\circ} \mathrm{C}$. Casein-coated wells served as a control for non-specific adhesion of rSsEno. The plates were washed three times with PBS, $\mathrm{pH} 7.3$, containing $0.05 \%(\mathrm{v} / \mathrm{v})$ Tween 20 (PBST), and $200 \mu 13 \%(\mathrm{w} / \mathrm{v})$ non-fat dried milk in PBST was added to each well to prevent non-specific binding. After $1 \mathrm{~h}$ at $37^{\circ} \mathrm{C}$, the wells were washed three times with PBST. Next, $100 \mu \mathrm{l}$ $5 \mu \mathrm{g} \mathrm{rSsEno} \mathrm{ml}^{-1}$ was added and the plates were incubated for $2 \mathrm{~h}$ at $37^{\circ} \mathrm{C}$. After several washes with PBST, optimally diluted rabbit anti-SsEno IgG was added to each well and the plates were incubated for $1 \mathrm{~h}$ at $37^{\circ} \mathrm{C}$. The wells were washed three times with PBST and specific horseradish peroxidase-labelled IgG (Jackson Immunoresearch Laboratories) was added. Plates were then incubated for $1 \mathrm{~h}$ at $37^{\circ} \mathrm{C}$ with the secondary antibody and 3,3',5,5'-tetramethylbenzidine (Zymed) was used as the enzyme substrate according to the manufacturer's instructions. The reactions were stopped by adding $25 \mu \mathrm{H}_{2} \mathrm{SO}_{4}(0.5 \mathrm{M})$ and were read at $450 \mathrm{~nm}$ using a microplate reader (Uvmax; Molecular Devices).
Binding interactions between $\mathrm{rSsEno}(75 \mathrm{kDa})$ and fibronectin $(440 \mathrm{kDa})$ or plasminogen $(92 \mathrm{kDa})$ were also analysed in real-time using Biacore 2000/3000 instrumentation. Surface plasmon resonance (SPR) assays were performed on research-grade CM4 sensor chips at $25{ }^{\circ} \mathrm{C}$ using filtered $(0.2 \mu \mathrm{m})$ and degassed HBS-EP running buffer [10 mM HEPES, pH 7.4, $150 \mathrm{mM} \mathrm{NaCl}, 3 \mathrm{mM}$ EDTA, $0.005 \%$ (v/v) Tween 20]. Prior to all experiments, the purity of protein preparations $(>95 \%)$ was assessed by SDS-PAGE ( $12.5 \%$ polyacrylamide) under non-reducing and reducing [5\% (v/v) 2-mercaptoethanol] conditions, followed by Coomassie blue and silver staining. Protein concentrations were determined using the Lowry method (Lowry et al., 1951). The zwitterionic detergent Empigen was from Calbiochem; all other chemicals were of reagent-grade quality.

Fibronectin or plasminogen $\left(10 \mu \mathrm{g} \mathrm{ml}^{-1}\right.$ in $10 \mathrm{mM}$ sodium acetate, $\mathrm{pH} 4.5$ ) was immobilized to sensor chip surfaces using the Amine Coupling kit (Biacore) according to the manufacturer's recommendations. Similarly, corresponding reference surfaces were prepared in the absence of ligand addition. rSsEno (0-250 nM diluted in HBSEP) was injected in-tandem over reference (no ligand) and active [290 relative units (RU) fibronectin or $140 \mathrm{RU}$ plasminogen-immobilized] surfaces at $25 \mu \mathrm{l} \mathrm{min} \operatorname{mos}^{-1}(600 \mathrm{~s}$ association $+600 \mathrm{~s}$ dissociation). Surfaces were regenerated at $50 \mu \mathrm{lmin}^{-1}$ using two $30 \mathrm{~s}$ pulses of solution I [HBS-EP containing $1 \mathrm{M} \mathrm{NaCl}, 5 \mathrm{mM} \mathrm{NaOH}$, and $0.03 \%$ $(\mathrm{v} / \mathrm{v})$ Empigen] and solution II (HBS-EP), followed by EXTRACLEAN and RINSE procedures. Additional assay controls to test for binding specificity (BSA negative control), surface performance (consistent replicate injections), and lack of mass transport were performed as recommended by the manufacturer.

All binding data were double-referenced (Myszka, 1999) and representative of triplicate injections acquired from three independent trials. Due to the slow off-rates observed (less than $10 \%$ dissociation over $10 \mathrm{~min}$ ), it was not possible to evaluate the data using standard kinetic $\left(K_{\mathrm{A}}, K_{\mathrm{D}}\right)$ analysis in the BIAevaluation 4.1 software (Biacore). Instead, the steady-state binding responses were averaged (540-580 s plateau) and then analysed globally as a function of rSsEno concentration. For non-linear regression analysis $\left(R_{\mathrm{eq}}\right.$ versus $C$ ), overall affinity constants were derived by fitting the data to a steady-state binding model:

$R_{\mathrm{eq}}=\left(R_{\mathrm{max}} \cdot C\right) /\left(K_{\mathrm{D}}+C\right)$

where $R_{\mathrm{eq}}$ is the binding response at equilibrium, $R_{\max }$ is the maximal specific binding to the ligand surface, $C$ is the concentration of analyte in solution, and $K_{\mathrm{D}}$ is the equilibrium dissociation constant. For linear regression analysis, $K_{\mathrm{D}}$ values were derived as the negative inverse of the slope generated by Scatchard transformations $\left(R_{\mathrm{eq}} / C\right.$ versus $\left.R_{\mathrm{eq}}\right)$.

Lysine-dependent rSsEno binding to fibronectin and plasminogen. To address the importance of lysine residues in the binding of rSsEno with fibronectin, competition binding assays were performed using the lysine analogue $\varepsilon$-amino- $n$-caproic acid (EACA). Briefly, fibronectin and the positive control plasminogen $\left(10\right.$ and $1 \mu \mathrm{g} \mathrm{ml}^{-1}$, respectively) were incubated overnight at $4{ }^{\circ} \mathrm{C}$ in Maxisorp flatbottomed microtitre 96-well plates, and an ELISA was performed as described above using $20 \mu \mathrm{g}$ rSsEno $\mathrm{ml}^{-1}$ mixed with different concentrations of EACA (1, 5, 10 and $50 \mathrm{mM}$; Sigma) and rabbit antiSsEno IgG as a primary antibody. Binding of SsEno to fibronectin and plasminogen in the absence of EACA competitor was considered to be $100 \%$.

Role of SsEno in adhesion and invasion of S. suis to PBMEC. The porcine brain microvascular endothelial cell (PBMEC) line PBMEC/C1-2 (Teifel \& Friedl, 1996), already tested with S. suis in our laboratory (Vanier et al., 2004), was used. The adhesion assay to quantify total cell-associated (intracellular plus surface-adhered) bacteria was performed as described previously (Nizet et al., 1997; 
Vanier et al., 2004), with some modifications. Briefly, S. suis strain $166^{\prime}\left(10^{7}\right.$ c.f.u. $\left.\mathrm{ml}^{-1}\right)$ was incubated with equal concentrations of either normal rabbit IgG or purifed rabbit anti-SsEno IgG for $1 \mathrm{~h}$ at $37{ }^{\circ} \mathrm{C}$ in fresh cell culture medium without antibiotics. Confluent cell monolayers were inoculated with $1 \mathrm{ml}$ aliquots of either bacterial suspension. The plates were centrifuged $(800 \mathrm{~g})$ and incubated for $2 \mathrm{~h}$ at $37{ }^{\circ} \mathrm{C}$ with $5 \% \mathrm{CO}_{2}$. The monolayers were then washed vigorously five times to eliminate non-specific bacterial attachment, incubated for $10 \mathrm{~min}$ at $37{ }^{\circ} \mathrm{C}$ in the presence of $200 \mu \mathrm{l} 0.05 \%$ trypsin, $0.03 \%$ EDTA, and disrupted by scraping the bottom of the well and by repeated pipetting to liberate cell-associated bacteria in the presence of ice-cold deionized water. Serial dilutions of this cell lysate were plated onto THB agar and incubated overnight at $37^{\circ} \mathrm{C}$, after which the bacteria were counted. The invasion assay to quantify intracellular bacteria was performed in a similar manner. However, to kill extracellular and surface-adhered bacteria after the initial infection period, cells were washed twice with $\mathrm{PBS}$, and $1 \mathrm{ml}$ cell culture medium containing $100 \mu \mathrm{g}$ gentamicin $\mathrm{ml}^{-1}$ and $5 \mu \mathrm{g}$ penicillin $\mathrm{G} \mathrm{ml}^{-1}$ (Sigma) was added to each well. After incubation for $1 \mathrm{~h}$ at $37{ }^{\circ} \mathrm{C}$ with $5 \% \mathrm{CO}_{2}$, monolayers were washed three times with PBS and processed as described above. Results obtained with bacteria incubated with normal rabbit IgG were considered as $100 \%$ adhesion or invasion.

Statistical analysis. All experiments were performed at least three times with samples in triplicate. All numerical data presented here are expressed as means \pm SD. Statistical significance was determined using Student's $t$ test. Differences were considered significant at $P \leqslant 0.05$

\section{RESULTS}

\section{Identification of a major S. suis fibronectin- binding protein of $52 \mathrm{kDa}$ as an $\alpha$-enolase}

Loading of $S$. suis lysates onto a fibronectin-affinity column resulted in the specific retention and elution of a predominant native $52 \mathrm{kDa}$ protein along with very few faint bands of additional proteins (Fig. 1). N-terminal amino acid sequencing of the $52 \mathrm{kDa}$ protein revealed the following 14 residues: MIITDVYAREVLDS. Comparison of this 14 aa $\mathrm{N}$-terminal sequence with the genomic sequence database of S. suis (http://www.sanger.ac.uk/ Projects/S_suis/) revealed $100 \%$ homology to the Nterminal region of a $1308 \mathrm{bp}$ ORF that encodes a putative 435 aa protein with a deduced molecular mass of $47.09 \mathrm{kDa}$. A protein BLAST search with the putative protein sequence in the NCBI sequence database (http:// www.ncbi.nlm.nih.gov/BLAST/) revealed significant homology to bacterial $\alpha$-enolases, a family of proteins involved in carbohydrate transport and metabolism. The identity between the putative $S$. suis protein and the $\alpha$ enolase from the closely related streptococci was more than $93 \%$ (data not shown). These results indicated that this $S$. suis $52 \mathrm{kDa}$ protein is most likely an $\alpha$-enolase (SsEno). Sequence analysis showed that the SsEno contains neither a signal peptide nor an LPXTG motif. This protein also does not contain choline-binding repeats. Immunoblot analysis confirmed the cross-reactivity of SsEno with polyclonal antibodies against Streptococcus pyogenes enolase (SEN; data not shown).

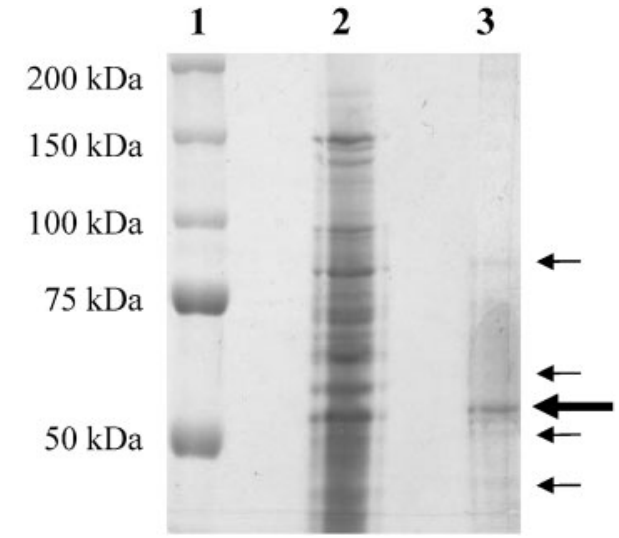

Fig. 1. Isolation of a $52 \mathrm{kDa}$ protein by fibronectin-affinity chromatography. S. suis lysate was applied to a fibronectincoupled $\mathrm{CNBr}$-activated Sepharose 4B column pre-equilibrated with $0.1 \mathrm{M} \mathrm{Tris} / \mathrm{HCl}$ buffer, $\mathrm{pH}$ 8.0. After washing, bound protein was eluted with $30 \mathrm{ml} 8 \mathrm{M}$ urea elution buffer. Elution profiles were analysed by SDS-PAGE (12.5\% polyacrylamide) with Coomassie blue staining. Lanes: 1, Molecular markers; $2, S$. suis total proteins; 3, S. suis fibronectin-binding proteins. Large arrow, $52 \mathrm{kDa}$ enolase; small arrows, other fibronectin-binding proteins.

\section{rSsEno has enolase activity}

rSsEno was successfully cloned and expressed as a Histagged fusion protein of $\sim 75 \mathrm{kDa}$ in E. coli. The higher molecular mass of the cloned protein compared with the native protein $(52 \mathrm{kDa})$ is due to the $\mathrm{N}$-terminal sequence of about $25 \mathrm{kDa}$ containing the His-tag added by the plasmid. The recombinant protein was purified to homogeneity $(>95 \%)$ using HisTrap affinity chromatography (Fig. 2), and it presents cross-reactivity identical to that of the native protein, as shown by immunoblot with antibodies against SEN (results not shown). To confirm that the fibronectin-binding protein candidate was an $\alpha$ enolase, its activity was assayed in a coupled enzyme assay for enolase. Similarly to the positive control used, we demonstrated that the recombinant protein was able to convert $\mathrm{NADH}$ to $\mathrm{NAD}$, resulting in a change in $A_{340}$ (Fig. 3). This indicated that pyruvate was converted to lactate and $\mathrm{NADH}$ by lactate dehydrogenase, confirming the conversion of phosphoglycerate to phosphoenolpyruvate by $\alpha$-enolase, and finally to pyruvate in the presence of externally provided pyruvate kinase and ADP in a sequential manner.

\section{SsEno has fibronectin- and plasminogen-binding activities}

Prior to all ELISA and SPR analyses, the purity ( $>95 \%)$ of commercially available fibronectin and plasminogen (data not shown) and rSsEno preparations was determined by scanning densitometry of the protein on an SDS-PAGE gel stained with Coomassie blue (data not shown) and on a 


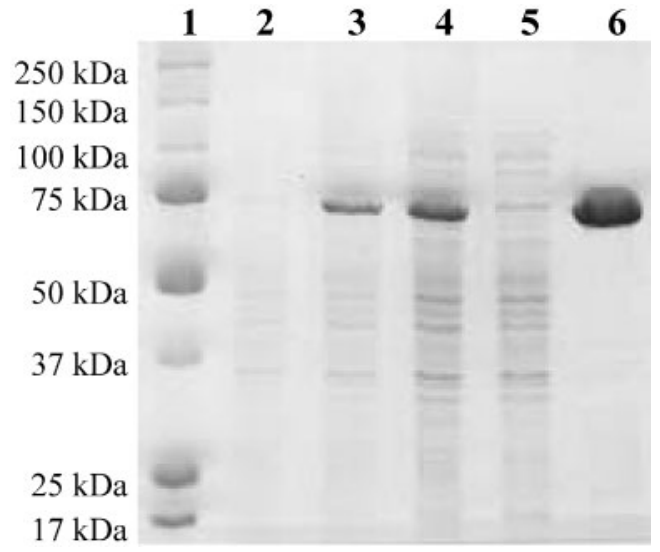

Fig. 2. Expression and purification of recombinant SsEno. The SsEno gene was cloned into $\mathrm{pET}-32 \mathrm{a}$, which was introduced into E. coli BI21DE3 for IPTG-inducible expression of rSsEno (recombinant $S$. suis enolase). The His-tagged fusion protein was purified by chromatography on a HisTrap column according to the manufacturer's protocols. Lanes: 1, molecular mass markers; 2, non-induced E. coli; 3, induced E. coli; 4, French press control; 5, HisTrap non-adhered proteins; 6, HisTrap-purified SsEno.

silver-stained SDS-PAGE gel (Fig. 4a). Qualitatively, ELISA assays provided the initial demonstration that rSsEno interacts specifically with immobilized fibronectin and plasminogen (positive control) in a saturable, dosedependent manner (Fig. 4b, c). Under similar assay conditions, rSsEno failed to interact with immobilized casein used as a negative control (Fig. 4d). Competition experiments with the lysine analogue EACA showed lysinedependent interactions between rSsEno and fibronectin or plasminogen (Fig. 5). Notably, the inhibition of rSsEno binding by EACA was more pronounced with plasminogen than with fibronectin.

To complement the ELISA datasets with more detailed quantitative kinetic analysis, rSsEno binding interactions were also examined in real-time by SPR. Specific, dosedependent binding of rSsEno to immobilized fibronectin or plasminogen (positive control) was characterized by slow association $\left(K_{\mathrm{A}}\right)$ and slow dissociation $\left(K_{\mathrm{D}}\right)$ rates in both cases (Fig. 6a, c). Under similar assay conditions, BSA failed to interact with the immobilized surfaces used as a negative control (data not shown). Due to the slow offrates observed for rSsEno binding to fibronectin or plasminogen (less than $10 \%$ dissociation over $10 \mathrm{~min}$ ), it was not possible to evaluate the data using standard kinetic $\left(K_{\mathrm{A}}, K_{\mathrm{D}}\right)$ analysis in the BIAevaluation 4.1 software (Biacore). Instead, steady-state binding responses were averaged at the end of the association phase (540-580 s plateau) and then analysed globally as a function of rSsEno concentration. Linear and non-linear regression analyses (Fig. 6b, d) indicated similarly high-affinity interactions between SsEno and fibronectin $\left(K_{\mathrm{D}} \sim 21 \mathrm{nM}\right)$ and SsEno and plasminogen $\left(K_{\mathrm{D}} \sim 14 \mathrm{nM}\right)$.

\section{SsEno is present both intracellularly and at the bacterial surface}

Western blot results showed that SsEno is present in S. suis supernatant, cell wall and cytoplasmic fractions, with negligible amounts in the membrane fraction (data not shown). Immunoelectron microscopy using the same antibody confirmed the surface location of SsEno on $S$. suis (strain 166') cells (Fig. 7a). In contrast, no labelling was observed for the negative control (Fig. 7b). (a)

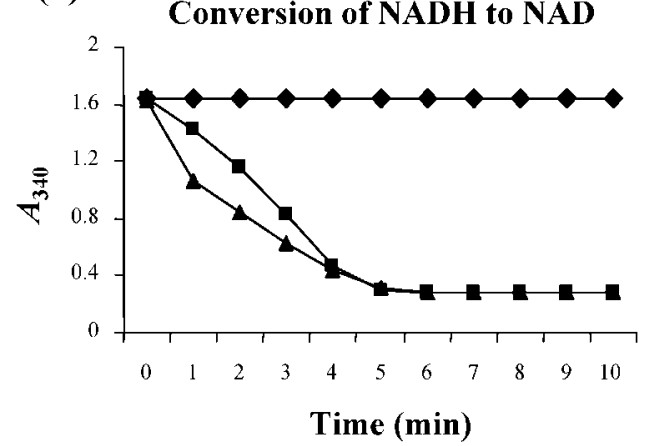

(b)

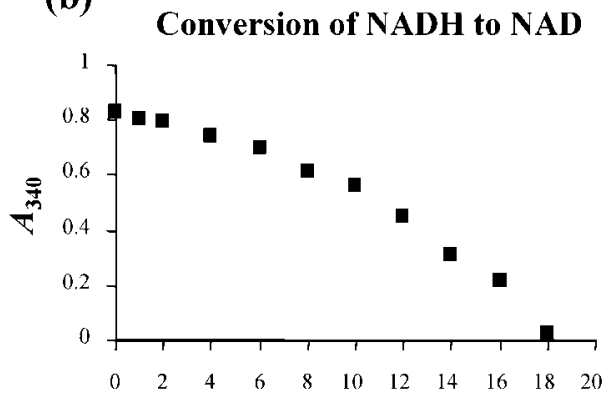

Purified SsEno added $(\mu \mathrm{g})$

Fig. 3. Enolase activity assay of recombinant SsEno. $\alpha$-Enolase activity of the purified rSsEno was measured using a coupled enzyme assay catalysing 2-phosphoglycerate to phosphoenolpyruvate, as described in Methods. (a) Enzyme kinetics of the purified rSsEno were determined, using $1 \mu \mathrm{g}$ purified $\mathrm{rSsEno}(\boldsymbol{\Delta})$ or $1 \mu \mathrm{g}$ rabbit muscle enolase as positive control ( $\boldsymbol{\square})$ as the enzyme source. As a negative control, enolase was omitted ( $\boldsymbol{(})$. $\alpha$-Enolase activity of SsEno was also measured as described in Methods. (b) Complementary assay, in which different SsEno concentrations were used as enzyme source and the enzyme activity in the reaction mixture was measured at the end of $1 \mathrm{~min}$ incubation. Data represent results of one individual experiment. The enolase assay was done at least in triplicate. 
(a)

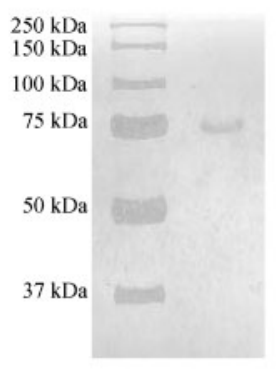

(c)

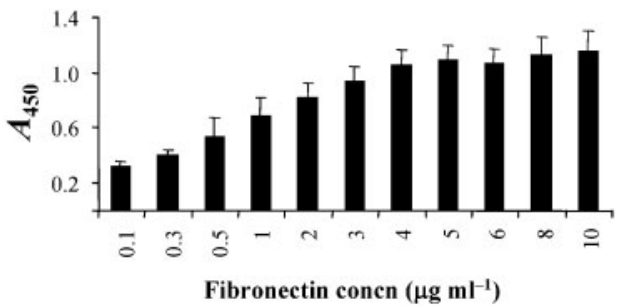

(b)

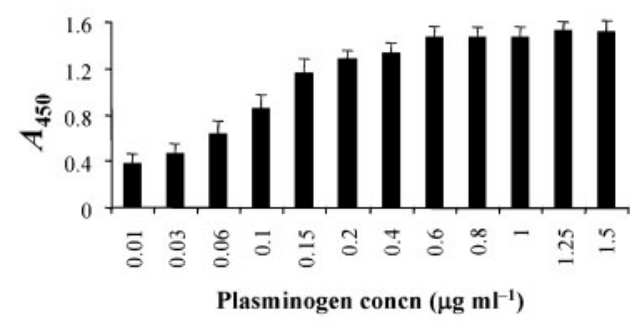

(d)

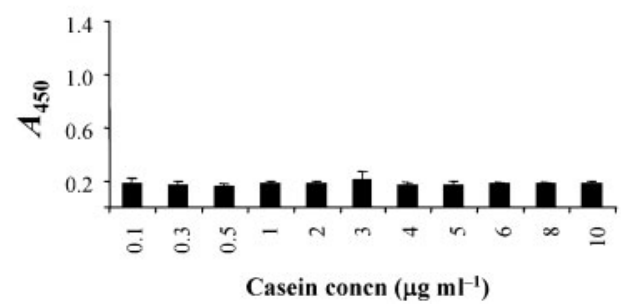

Fig. 4. Qualitative binding of SsEno to fibronectin and plasminogen. (a) Silver nitrate staining of SsEno used for ELISA and SPR tests. (b-d) Representative ELISA analysis for the binding of SsEno ( $5 \mu \mathrm{g} \mathrm{ml}^{-1}$ ) to immobilized plasminogen (b), fibronectin (c) and casein ( $d$, negative control). Binding was evaluated after $2 \mathrm{~h}$. Data are expressed as mean $\pm S D$ of at least three experiments with samples in triplicate.

\section{SsEno participates in S. suis adhesion to and invasion of PBMEC}

To evaluate the potential role of SsEno in S. suis adhesion to and invasion of PBMEC, bacterial SsEno was blocked with affinity-purified rabbit anti-SsEno IgG. As shown in Fig. 8, anti-SsEno IgG treatment decreased the adhesion of S. suis serotype 2 strain $166^{\prime}$ to PBMEC to $\sim 46 \%$ compared with the control. The invasion of PBMEC by

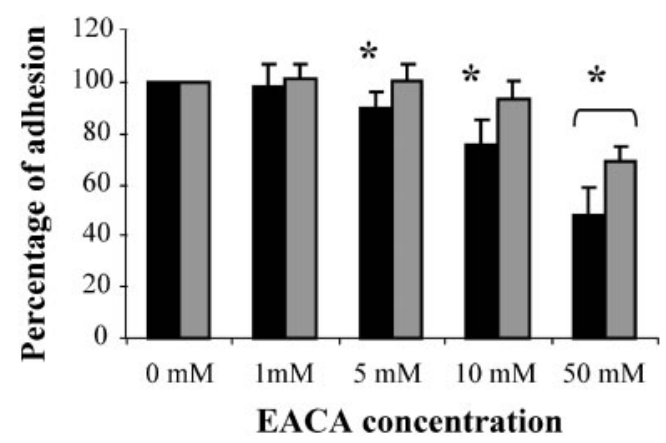

Fig. 5. Lysine-dependent binding of SsEno to fibronectin and plasminogen. Different EACA concentrations were used to evaluate the role of the lysine residues in the binding of SsEno to plasminogen (black bars) and fibronectin (grey bars) by ELISA, as described in Methods. ${ }^{*} P<0.05$ in comparison with the level of adhesion without competitor (considered to be $100 \%$ adhesion). Data are expressed as mean \pm SD of at least three experiments with samples in triplicate.
S. suis was also decreased to $\sim 79 \%$ compared with the control.

\section{DISCUSSION}

Over the past 15 years, S. suis infections have emerged as a major problem for the swine industry worldwide, and it is also considered an important zoonotic agent (Higgins \& Gottschalk., 2005; Tang et al., 2006; Ye et al., 2006). The pathogen is able to spread systemically from the nasopharynx, resulting in either general septicaemia or infections of specific organs (e.g. endocarditis, meningitis and arthritis), followed frequently by death (Gottschalk \& Segura, 2000; Madsen et al., 2002). The palatine and pharyngeal tonsils are both potential portals of entry for S. suis, although the mechanisms and virulence factors that enable the pathogen to disseminate throughout the animal and colonize different tissues are not well understood.

Adhesion of pathogens to host tissues is a critical early step in the process of infection. The ability to bind to fibronectin is a characteristic that has been reported for many pathogens (Joh et al., 1999). As fibronectin plays an important role in diverse normal physiological processes, its targeting appears to be an example of the exploitation of a host cell process in the establishment, maintenance or dissemination of infection (Knodler et al., 2001). Although a previously reported FBPS has been described for S. suis (de Greeff et al., 2002), a fbps mutant has been shown to adhere to fibronectin at similar levels to those of the wild- 
(a)

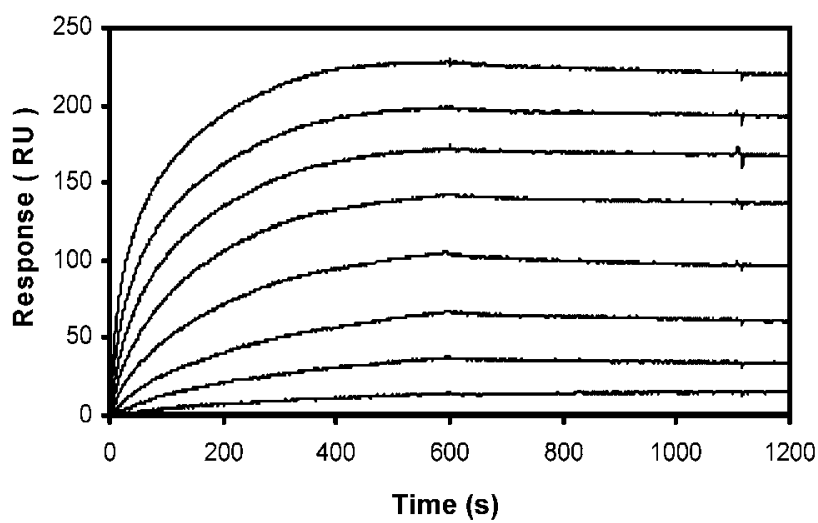

(c)

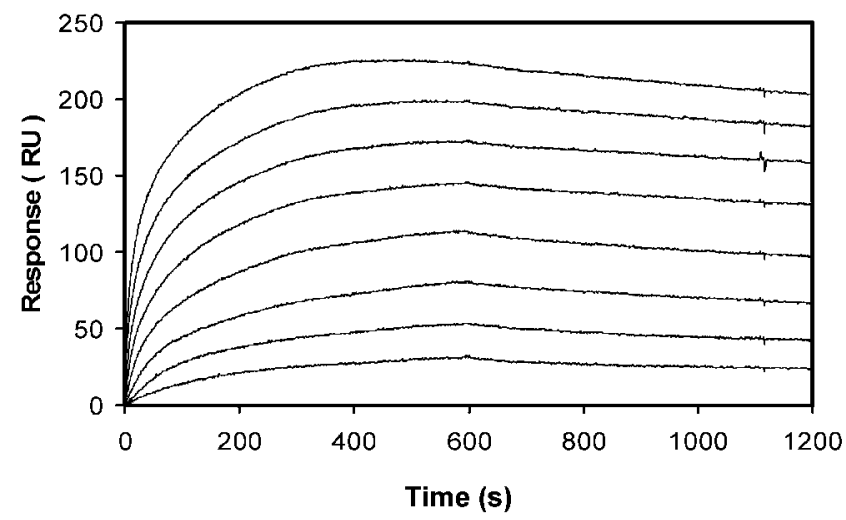

(b)

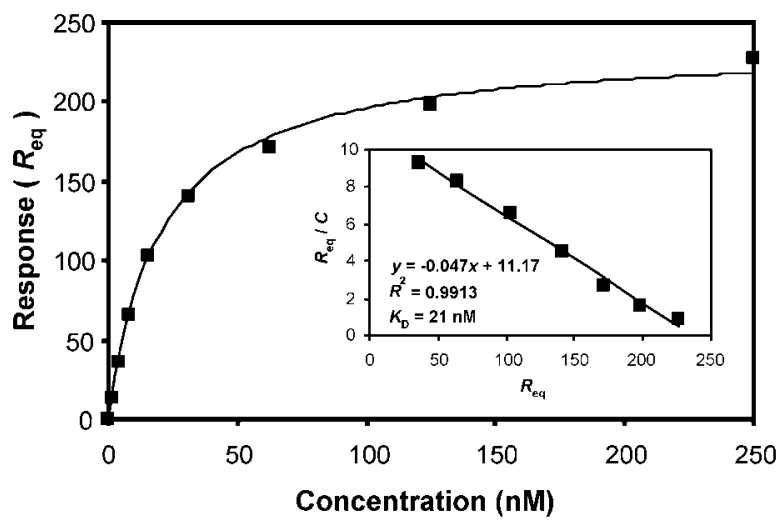

(d)

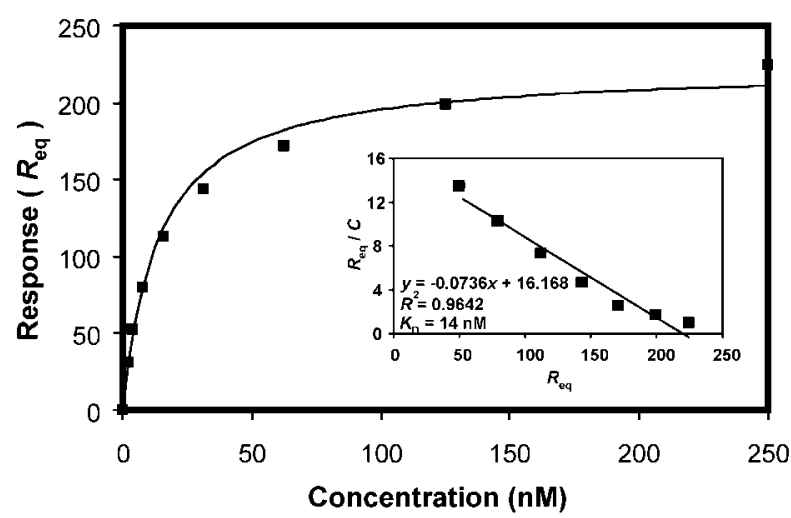

Fig. 6. Quantitative binding of SsEno to fibronectin and plasminogen. Representative SPR analysis of rSsEno (0, 3.9, 7.8, 15.6, $31.3,62.5,125$ and $250 \mathrm{nM}$ ) injected (600 s association + $600 \mathrm{~s}$ dissociation) over amine-coupled fibronectin [290 RU; (a)] or plasminogen [140 RU; (c)] at $25 \mu \mathrm{min}^{-1}$. Equilibrium binding responses $\left(R_{\mathrm{eq}}\right)$ were averaged $(540-580 \mathrm{~s})$ and then evaluated according to the steady-state affinity model $\left[R_{\text {eq }}\right.$ versus concentration $(C)$; fibronectin (b); plasminogen (d)]. Subsequent Scatchard transformations ( $R_{\text {eq }} / C$ versus $R_{\text {eq }}$; insets) indicate similar high-affinity interactions between SsEno and fibronectin $\left(K_{\mathrm{D}} \sim 21 \mathrm{nM}\right)$ and SsEno and plasminogen $\left(K_{\mathrm{D}} \sim 14 \mathrm{nM}\right)$.

type parent strain (Vanier et al., 2007a), suggesting that other major adhesins are also involved.

In the present study, using fibronectin-coupled affinity chromatography, we have identified a predominant $S$. suis fibronectin-binding native protein of $52 \mathrm{kDa}$. Subsequently, the protein was identified as an $\alpha$-enolase based upon the high similarity of its sequence with other bacterial enolases $(93 \%$ with other streptococcal $\alpha$ enolases) and our biochemical tests confirmed enolase activity. Furthermore, SsEno is also cross-reactive with anti-SEN antiserum, which has been shown to react with $\alpha$ enolase-like molecules on streptococci (Pancholi \& Fischetti, 1998). This is the first time, to our knowledge, that the fibronectin-binding activity of an $\alpha$-enolase has been described. These results were confirmed using rSsEno cloned and expressed in the present study. In fact, Antikainen et al. (2007) have recently reported that $\alpha$-enolases from other Gram-positive bacteria, such as $S$. pyogenes, Streptococcus pneumoniae, Staphylococcus aureus, Lactobacillus crispatus and Lactobacillus johnsonii, do not possess the ability to bind to fibronectin. Thus, the interaction of enolase with fibronectin may serve different purposes in $S$. suis from those in the pathogenic and commensal bacteria studied in that work. Interestingly, after a complete proteome analysis, Jing et al. (2008) have recently proposed the enolase as one of two putative virulence-associated factors for S. suis serotype 2. However, the hypothetical plasminogen binding of the S. suis enolase (based on earlier studies performed with other streptococci) is proposed in that study as the mechanism by which this virulence factor participates in the pathogenesis of the infection caused by this pathogen.

To possess fibronectin-binding activity, it was anticipated that SsEno should be exposed at the surface of bacteria. 


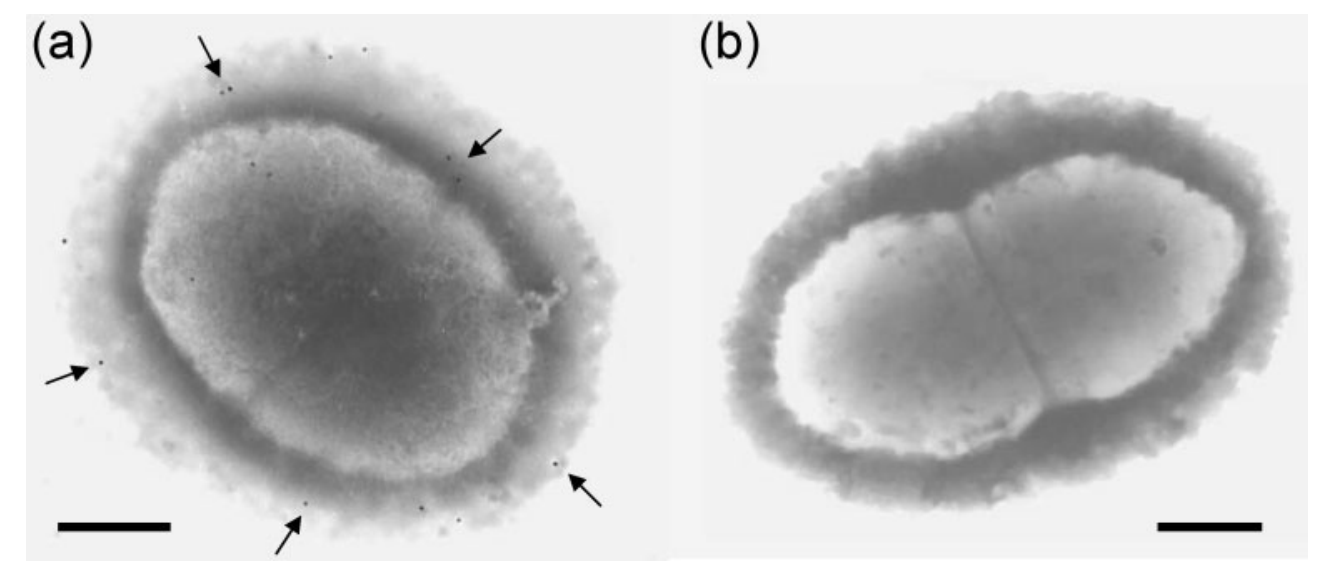

Fig. 7. Surface localization of SsEno on S. suis. (a) Immunogold electron microscopic detection of the location of SsEno (×45000 magnification) on S. suis pretreated with rabbit anti-SsEno lgG, followed by colloidal-gold-labelled anti-donkey lgG (gold particle sizes are $12 \mathrm{~nm}$; indicated by the arrows). (b) Normal rabbit lgG used as a negative control. Bars, $250 \mathrm{~nm}$.
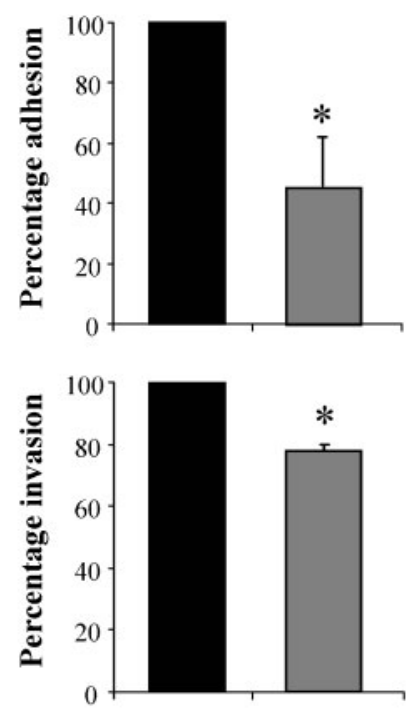

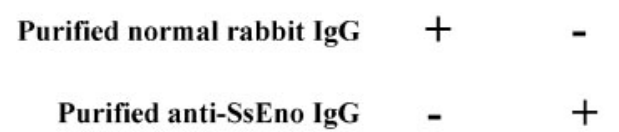

Fig. 8. Decrease in the adhesion to and invasion of PBMEC by $S$. suis strain SS166' caused by antibodies against SsEno. S. suis strain $166^{\prime}$ was preincubated with the appropriate concentrations $(10 \mu \mathrm{g})$ of antibodies at $37^{\circ} \mathrm{C}$ for $60 \mathrm{~min}$ prior to infection. Adhesion was determined after exposure of PBMEC to $S$. suis $\left(10^{7}\right.$ c.f.u. $\left.\mathrm{ml}^{-1}\right)$, followed by extensive washing of non-adherent bacteria and cell lysis to obtain $S$. suis viable plate counts on THB. Invasion was determined as described above, except that after washing the bacteria, the cells were exposed to antibiotics to kill extracellular bacteria. ${ }^{*} P<0.05$ in comparison with the level of adhesion/invasion with control antibodies (considered to be $100 \%$ adhesion/invasion).
Fractionation of S. suis strain $166^{\prime}$ showed that SsEno is present in all the subcellular components, including the bacterial surface, as demonstrated for other pathogens, including S. pyogenes (Pancholi \& Fischetti, 1998). ELISA tests carried out with whole bacteria and anti-SsEno also showed a clear recognition of this protein at the bacterial surface (M. Esgleas and others, unpublished observations). Electron microscopy corroborated this surface localization and also showed that SsEno is present at the surface in low abundance, a feature that has also been described for $S$. pneumoniae enolase (Kolberg et al., 2006). Moreover, such a relatively low amount has proven sufficient for contributing to bacterial pathogenesis (Bergmann et al., 2003). Interestingly, in addition to serotype 2 , all reference strains of other serotypes of $S$. suis were found to express surface-exposed SsEno (M. Esgleas and others, unpublished observations). The mechanisms by which this protein is translocated to the bacterial surface are still unknown. In fact, the amino acid sequence of SsEno shows the absence of an N-terminal signal sequence and of an anchor C-terminus LPXTG motif. It has been demonstrated that this LPXTG motif is necessary for the translocation of proteins to the bacterial surface because it is recognized by a transpeptidase (sortase A) that cleaves between the Thr and Gly residues (Schneewind et al., 1995). Once cleaved, the carboxyl group of the Thr residue is linked to a free amino group of a branch peptide within the peptidoglycan cell wall, permitting the anchorage of these LPXTG-containing proteins to the bacterial cell wall (Schneewind et al., 1995). Interestingly, we have shown recently that disruption of the srtA gene in S. suis results in only a slight decrease in adhesion to fibronectin (Vanier et al., 2007b), confirming that anchorless (such as the enolase) rather than LPXTG-motif proteins play a major role in the adhesion to this ECM protein.

To date, the major function described for surface enolases is a strong plasminogen-binding activity (Pancholi \& 
Fischetti, 1998; Pancholi, 2001). In this study we confirmed that, in addition to a previously described GAPDH (Jobin et al., 2004), SsEno is also an S. suis plasminogen-binding protein. Although it has been demonstrated recently that enolases of other Gram-positive bacteria bind to laminin and/or collagen (Antikainen et al., 2007), we could not identify any adhesion activity of rSsEno to either of these two proteins (M. Esgleas and others, unpublished observations). Interestingly, in addition to the fibronectin and plasminogen-binding activities of the rSsEno, a heat-shock protein activity [as described previously for other enolases (Iida \& Yahara, 1985; Prasad et al., 2003)] and an IgGbinding property of this protein have also been observed (M. Esgleas and others, unpublished observations).

The binding of purified rSsEno to both plasminogen and fibronectin was demonstrated qualitatively by ELISA and quantitatively by SPR. Interestingly, a higher amount of fibronectin, compared with that used for plasminogen, had to be used in the ELISA test to obtain similar absorbances. Although this might be interpreted as a significantly higher affinity (which was not confirmed by SPR), this is not the case. The ELISA test was used in this study as a qualitative test, since plasminogen and fibronectin have different molecular masses and their attachment to plastic wells may vary with the conditions used for the ELISA. In particular, SPR analysis indicated clearly that rSsEno interactions with plasminogen and fibronectin were of similarly high affinity ( $K_{\mathrm{D}} \sim 14 \mathrm{nM}$ and $\sim 21 \mathrm{nM}$, respectively). High-affinity binding between SsEno and fibronectin is consistent with other bacterial fibronectin-binding adhesins, including F1 and F2 of S. pyogenes (Ensenberger et al., 2001; Kreikemeyer et al., 2004). These results support our hypothesis that the specific interaction between SsEno and fibronectin is biologically significant. Both ELISA and SPR also demonstrated, as described for other pathogens, that the interaction between rSsEno and plasminogen is specific, suggesting that this interaction is also a biologically significant event. This provides further evidence to support the earlier finding that bacterial enolases interact with plasminogen with high affinity (Antikainen et al., 2007; Kinnby et al., 2008). In fact, the low nanomolar affinity constant between SsEno and plasminogen obtained in this study agrees with values obtained for the adhesion to plasminogen of enolases from S. pyogenes (Pancholi \& Fischetti, 1998) and S. pneumoniae (Bergmann et al., 2003).

Plasminogen-binding interactions were originally described as being mediated by recognition of the Cterminal lysine residues of eukaryotic and prokaryotic enolases by the lysine-binding sites of plasminogen (Redlitz et al., 1995). Later, Bergmann et al. (2003) identified another internal plasminogen-binding site in the pneumococcal enolase (FYDKERKVY) that is located on the outer surface of the octameric molecule (Ehinger et al., 2004), in which the lysines and glutamic acid are important for plasminogen binding (Bergmann et al., 2005). Importantly, SsEno possesses both plasminogen-binding motifs (M. Esgleas and others, unpublished observations), which could explain the similar nanomolar affinity constant of both S. pneumoniae (Bergmann et al., 2003) and S. suis enolase to plasminogen. Binding of plasminogen to S. suis enolase is inhibited by the lysine analogue EACA, which indicates involvement of lysine residues in this binding activity. Interestingly, SsEno adhesion to fibronectin is also decreased by EACA, indicating that lysine residues are also important for its fibronectin-binding activity. However, further research is required to determine the specific domain(s) and the individual amino acids that participate in the binding of SsEno to fibronectin.

As mentioned above, the pathogenesis of $S$. suis infection is poorly understood at present. Once in the bloodstream, $S$. suis resists phagocytosis and killing by neutrophils and monocytes (Chabot-Roy et al., 2006; Charland et al., 1998; Segura et al., 1998; Smith et al., 1999). In the event that $S$. suis fails to cause acute fatal septicaemia, bacteria are able to reach the CNS via different mechanisms that are only partially elucidated, such as adhesion to, with or without toxicity, and invasion of brain microvascular endothelial cells (BMEC) (Benga et al., 2005; Charland et al., 2000; Vanier et al., 2004) and/or choroid plexus epithelial cells (Tenenbaum et al., 2005, 2006). In fact, interactions of $S$. suis with both fibronectin and plasminogen may play a role in some of these mechanisms. For example, fibronectinbinding proteins of streptococci and staphylococci have been reported to mediate bacterial adhesion to and invasion of host cells (Oehmcke et al., 2004; Talay et al., 2000; Valentin-Weigand et al., 1993). In the case of S. suis, it has been demonstrated recently that $S$. suis adhesion to and intracellular invasion of PBMEC increases more than 500 and $700 \%$, respectively, when bacteria are precoated with fibronectin (Vanier et al., 2007a). Although the mechanisms by which fibronectin-binding proteins trigger the internalization of bacteria by mammalian cells are not completely understood, it has been suggested that binding of fibronectin to a host integrin might initiate a complex cascade of cell signalling that leads to reorganization of cytoskeletal components and consequent internalization of the bacteria (Schwarz-Linek et al., 2004). Accordingly, S. suis invasion of endothelial cells is actin-dependent (Vanier et al., 2004). In the present study, a role for SsEno in adhesion to and invasion of these cells has been shown by blocking the protein using affinity-purified anti-SsEno IgG. Since these studies have been carried out with cell monolayers, the fibronectin-binding activity of SsEno might explain this inhibition. It might be hypothesized that $S$. suis enolase is an important receptor for plasma fibronectin, which may increase bacterial adhesion to host cells. Another mechanism involved in adhesion of S. suis enolase to cellular fibronectin present in endothelial cells cannot be completely ruled out. However, further studies have to be carried out to demonstrate such an interaction. Although no increase in adhesion to/invasion of PBMEC was observed when increased concentrations of plasminogen were added ( $M$. Esgleas and others, unpublished observations), a role for the plasminogen-binding activity 
of SsEno (and GAPDH) in vivo cannot be discounted. In fact, it has also been demonstrated that plasminogen adhered to $S$. suis is able to acquire plasmin activity when co-incubated with human BMEC (Jobin et al., 2005). As a consequence of plasminogen activation on bacterial surfaces, bacteria become armed with the broad-substrate-spectrum proteolytic potential of plasmin that is not susceptible to regulation by host-derived inhibitors (Lottenberg et al., 1994). The capture of plasminogen by adhesins such as SsEno and GAPDH and its conversion to plasmin has been described for other pathogens (Antikainen et al., 2007; Kinnby et al., 2008) and can be used to facilitate bacterial penetration through biological membranes such as the blood-brain barrier, and therefore could represent an important determinant of virulence. The role of SsEno in studies using endothelial-cell monolayers in a trans-well system is currently under investigation in our laboratory.

In conclusion, we have demonstrated for the first time, to our knowledge, that $S$. suis $\alpha$-enolase has not only a high affinity for plasminogen, as demonstrated for other bacterial enolases, but also a similarly high affinity for fibronectin. Moreover, we have shown that this protein is a novel S. suis adhesin that participates in bacterial adhesion to and invasion of endothelial cells. Our results indicate that SsEno of this human and porcine pathogen could be a key molecule in pathogenesis by facilitating bacterial interactions with host cells. This is in agreement with two recent studies that describe $S$. suis enolase as an antigenic protein and a putative virulence factor of $S$. suis serotype 2 (Jing et al., 2008; Zhang \& Lu, 2007). Mutants with defective export of enolase to the surface of the bacteria may give some insights into the role of this adhesin in the pathogenesis of S. suis infection.

\section{ACKNOWLEDGEMENTS}

This work was supported by the Natural Sciences and Engineering Research Council of Canada (NSERC) grant 0680154280. Sheldon Biotechnology Centre is supported by a Research Resource Grant from the Canadian Institutes of Health Research (CIHR). Canada Foundation for Innovation (CFI) provided infrastructure for the SPR instrumentation. We thank Dr Vijay Pancholi (The International Center for Public Health, Newark, NJ) for providing the polyclonal antibodies against SEN. We also thank M. Mourez and N. Fittipaldi for useful discussions.

\section{REFERENCES}

Al-Numani, D., Segura, M., Dore, M. \& Gottschalk, M. (2003). Upregulation of ICAM-1, CD11a/CD18 and CD11c/CD18 on human THP-1 monocytes stimulated by Streptococcus suis serotype 2. Clin Exp Immunol 133, 67-77.

Antikainen, J., Kuparinen, V., Lahteenmaki, K. \& Korhonen, T. K. (2007). Enolases from Gram-positive bacterial pathogens and commensal lactobacilli share functional similarity in virulenceassociated traits. FEMS Immunol Med Microbiol 51, 526-534.
Baums, C. G., Kaim, U., Fulde, M., Ramachandran, G., Goethe, R. \& Valentin-Weigand, P. (2006). Identification of a novel virulence determinant with serum opacification activity in Streptococcus suis. Infect Immun 74, 6154-6162.

Benga, L., Friedl, P. \& Valentin-Weigand, P. (2005). Adherence of Streptococcus suis to porcine endothelial cells. J Vet Med B Infect Dis Vet Public Health 52, 392-395.

Bergmann, S., Rohde, M., Chhatwal, G. S. \& Hammerschmidt, S. (2001). $\alpha$-Enolase of Streptococcus pneumoniae is a plasmin(ogen)binding protein displayed on the bacterial cell surface. Mol Microbiol 40, 1273-1287.

Bergmann, S., Wild, D., Diekmann, O., Frank, R., Bracht, D., Chhatwal, G. S. \& Hammerschmidt, S. (2003). Identification of a novel plasmin(ogen)-binding motif in surface displayed $\alpha$-enolase of Streptococcus pneumoniae. Mol Microbiol 49, 411-423.

Bergmann, S., Rohde, M., Preissner, K. T. \& Hammerschmidt, S. (2005). The nine residue plasminogen-binding motif of the pneumococcal enolase is the major cofactor of plasmin-mediated degradation of extracellular matrix, dissolution of fibrin and transmigration. Thromb Haemost 94, 304-311.

Berthelot-Herault, F., Gottschalk, M., Morvan, H. \& Kobisch, M. (2005). Dilemma of virulence of Streptococcus suis: Canadian isolate 89-1591 characterized as a virulence strain using a standardized experimental model in pigs. Can J Vet Res 69, 236-240.

Brassard, J., Gottschalk, M. \& Quessy, S. (2001). Decrease of the adhesion of Streptococcus suis serotype 2 mutants to embryonic bovine tracheal cells and porcine tracheal rings. Can J Vet Res 65, 156-160.

Brassard, J., Gottschalk, M. \& Quessy, S. (2004). Cloning and purification of the Streptococcus suis serotype 2 glyceraldehyde-3phosphate dehydrogenase and its involvement as an adhesin. Vet Microbiol 102, 87-94.

Chabot-Roy, G., Willson, P., Segura, M., Lacouture, S. \& Gottschalk, M. (2006). Phagocytosis and killing of Streptococcus suis by porcine neutrophils. Microb Pathog 41, 21-32.

Charland, N., Harel, J., Kobisch, M., Lacasse, S. \& Gottschalk, M. (1998). Streptococcus suis serotype 2 mutants deficient in capsular expression. Microbiology 144, 325-332.

Charland, N., Nizet, V., Rubens, C. E., Kim, K. S., Lacouture, S. \& Gottschalk, M. (2000). Streptococcus suis serotype 2 interactions with human brain microvascular endothelial cells. Infect Immun 68, 637-643.

de Greeff, A., Buys, H., Verhaar, R., Dijkstra, J., van Alphen, L. \& Smith, H. E. (2002). Contribution of fibronectin-binding protein to pathogenesis of Streptococcus suis serotype 2. Infect Immun 70, 1319-1325.

Ehinger, S., Schubert, W. D., Bergmann, S., Hammerschmidt, S. \& Heinz, D. W. (2004). Plasmin(ogen)-binding $\alpha$-enolase from Streptococcus pneumoniae: crystal structure and evaluation of plasmin(ogen)-binding sites. J Mol Biol 343, 997-1005.

Ensenberger, M. G., Tomasini-Johansson, B. R., Sottile, J., Ozeri, V., Hanski, E. \& Mosher, D. F. (2001). Specific interactions between F1 adhesin of Streptococcus pyogenes and N-terminal modules of fibronectin. J Biol Chem 276, 35606-35613.

Esgleas, M., Lacouture, S. \& Gottschalk, M. (2005). Streptococcus suis serotype 2 binding to extracellular matrix proteins. FEMS Microbiol Lett 244, 33-40.

Gottschalk, M. \& Segura, M. (2000). The pathogenesis of the meningitis caused by Streptococcus suis: the unresolved questions. Vet Microbiol 76, 259-272.

Higgins, R. \& Gottschalk, M. (2005). Streptococcal diseases. In Diseases of Swine, 9th edn, pp. 769-783. Edited by B. E. Straw, J. J. 
Zimmerman, S. D’Allaire \& D. J. Taylor. Ames, IA: Iowa State University Press.

Hill, J. E., Gottschalk, M., Brousseau, R., Harel, J., Hemmingsen, S. M. \& Goh, S. H. (2005). Biochemical analysis, cpn60 and 16S rDNA sequence data indicate that Streptococcus suis serotypes 32 and 34, isolated from pigs, are Streptococcus orisratti. Vet Microbiol 107, 63-69.

lida, H. \& Yahara, I. (1985). Yeast heat-shock protein of $M_{\mathrm{r}} 48,000$ is an isoprotein of enolase. Nature 315, 688-690.

Jing, H. B., Yuan, J., Wang, J., Yuan, Y., Zhu, L., Liu, X. K., Zheng, Y. L., Wei, K. H., Zhang, X. M. \& other authors (2008). Proteome analysis of Streptococcus suis serotype 2. Proteomics 8, 333-349.

Jobin, M. C., Brassard, J., Quessy, S., Gottschalk, M. \& Grenier, D. (2004). Acquisition of host plasmin activity by the swine pathogen Streptococcus suis serotype 2. Infect Immun 72, 606-610.

Jobin, M. C., Fortin, J., Willson, P. J., Gottschalk, M. \& Grenier, D. (2005). Acquisition of plasmin activity and induction of arachidonic acid release by Streptococcus suis in contact with human brain microvascular endothelial cells. FEMS Microbiol Lett 252, 105-111.

Joh, D., Wann, E. R., Kreikemeyer, B., Speziale, P. \& Hook, M. (1999). Role of fibronectin-binding MSCRAMMs in bacterial adherence and entry into mammalian cells. Matrix Biol 18, 211-223.

Jolodar, A., Fischer, P., Bergmann, S., Buttner, D. W., Hammerschmidt, S. \& Brattig, N. W. (2003). Molecular cloning of an $\alpha$-enolase from the human filarial parasite Onchocerca volvulus that binds human plasminogen. Biochim Biophys Acta 1627, 111-120.

Jong, A. Y., Chen, S. H., Stins, M. F., Kim, K. S., Tuan, T. L. \& Huang, S. H. (2003). Binding of Candida albicans enolase to plasmin(ogen) results in enhanced invasion of human brain microvascular endothelial cells. J Med Microbiol 52, 615-622.

Kinnby, B., Booth, N. A. \& Svensater, G. (2008). Plasminogen binding by oral streptococci from dental plaque and inflammatory lesions. Microbiology 154, 924-931.

Knodler, L. A., Celli, J. \& Finlay, B. B. (2001). Pathogenic trickery: deception of host cell processes. Nat Rev Mol Cell Biol 2, 578-588.

Kolberg, J., Aase, A., Bergmann, S., Herstad, T. K., Rodal, G., Frank, R., Rohde, M. \& Hammerschmidt, S. (2006). Streptococcus pneumoniae enolase is important for plasminogen binding despite low abundance of enolase protein on the bacterial cell surface. Microbiology 152, 1307-1317.

Kreikemeyer, B., Oehmcke, S., Nakata, M., Hoffrogge, R. \& Podbielski, A. (2004). Streptococcus pyogenes fibronectin-binding protein F2: expression profile, binding characteristics, and impact on eukaryotic cell interactions. J Biol Chem 279, 15850-15859.

Kreis, T. \& Vale, R. (1993). Guidebook to the Extracellular Matrix and Adhesion Proteins. Oxford: Oxford University Press.

Lalonde, M., Segura, M., Lacouture, S. \& Gottschalk, M. (2000). Interactions between Streptococcus suis serotype 2 and different epithelial cell lines. Microbiology 146, 1913-1921.

Li, Y., Martinez, G., Gottschalk, M., Lacouture, S., Willson, P., Dubreuil, J., Jacques, M. \& Harel, J. (2006). Identification of a surface protein of Streptococcus suis and evaluation of its immunogenic and protective capacity in pigs. Infect Immun 74, 305-312.

Lottenberg, R., Minning-Wenz, D. \& Boyle, M. D. (1994). Capturing host plasmin(ogen): a common mechanism for invasive pathogens? Trends Microbiol 2, 20-24.

Lowry, O. H., Rosebrough, N. J., Farr, A. L. \& Randall, R. J. (1951). Protein measurement with the Folin phenol reagent. J Biol Chem 193, 265-275.

Lun, Z.-R., Wang, Q.-P., Chen, X.-G., Li, A.-X. \& Zhu, X.-Q. (2007). Streptococcus suis: an emerging zoonotic pathogen. Lancet Infect Dis $\mathbf{7}$, 201-209.
Madsen, L. W., Svensmark, B., Elvestad, K., Aalbaek, B. \& Jensen, H. E. (2002). Streptococcus suis serotype 2 infection in pigs: new diagnostic and pathogenic aspects. J Comp Pathol 126, 57-65.

Marks, D. B. (1998). Carbohydrate metabolism. In Biochemistry, pp. 135-192. Edited by D. B. Marks. Baltimore, MD: Lippincott Williams and Wilkins.

Miles, L. A., Dahlberg, C. M., Plescia, J., Felez, J., Kato, K. \& Plow, E. F. (1991). Role of cell-surface lysines in plasminogen binding to cells: identification of $\alpha$-enolase as a candidate plasminogen receptor. Biochemistry 30, 1682-1691.

Molkanen, T., Tyynela, J., Helin, J., Kalkkinen, N. \& Kuusela, P. (2002). Enhanced activation of bound plasminogen on Staphylococcus aureus by staphylokinase. FEBS Lett 517, 72-78.

Myszka, D. G. (1999). Improving biosensor analysis. J Mol Recognit 12, 279-284.

Nakajima, K., Hamanoue, M., Takemoto, N., Hattori, T., Kato, K. \& Kohsaka, S. (1994). Plasminogen binds specifically to $\alpha$-enolase on rat neuronal plasma membrane. J Neurochem 63, 2048-2057.

Nizet, V., Kim, K. S., Stins, M., Jonas, M., Chi, E. Y., Nguyen, D. \& Rubens, C. E. (1997). Invasion of brain microvascular endothelial cells by group B streptococci. Infect Immun 65, 5074-5081.

Norton, P. M., Rolph, C., Ward, P. N., Bentley, R. W. \& Leigh, J. A. (1999). Epithelial invasion and cell lysis by virulent strains of Streptococcus suis is enhanced by the presence of suilysin. FEMS Immunol Med Microbiol 26, 25-35.

Oehmcke, S., Podbielski, A. \& Kreikemeyer, B. (2004). Function of the fibronectin-binding serum opacity factor of Streptococcus pyogenes in adherence to epithelial cells. Infect Immun 72, 4302-4308.

Pancholi, V. (2001). Multifunctional $\alpha$-enolase: its role in diseases. Cell Mol Life Sci 58, 902-920.

Pancholi, V. \& Fischetti, V. A. (1998). $\alpha$-Enolase, a novel strong plasmin(ogen) binding protein on the surface of pathogenic streptococci. J Biol Chem 273, 14503-14515.

Pancholi, V., Fontan, P. \& Jin, H. (2003). Plasminogen-mediated group A streptococcal adherence to and pericellular invasion of human pharyngeal cells. Microb Pathog 35, 293-303.

Prasad, J., McJarrow, P. \& Gopal, P. (2003). Heat and osmotic stress responses of probiotic Lactobacillus rhamnosus HN001 (DR20) in relation to viability after drying. Appl Environ Microbiol 69, 917-925.

Quessy, S., Busque, P., Higgins, R., Jacques, M. \& Dubreuil, J. D. (1997). Description of an albumin binding activity for Streptococcus suis serotype 2. FEMS Microbiol Lett 147, 245-250.

Redlitz, A., Fowler, B. J., Plow, E. F. \& Miles, L. A. (1995). The role of an enolase-related molecule in plasminogen binding to cells. Eur $J$ Biochem 227, 407-415.

Schneewind, O., Fowler, A. \& Faull, K. F. (1995). Structure of the cell wall anchor of surface proteins in Staphylococcus aureus. Science 268, 103-106.

Schwarz-Linek, U., Hook, M. \& Potts, J. R. (2004). The molecular basis of fibronectin-mediated bacterial adherence to host cells. Mol Microbiol 52, 631-641.

Segura, M. \& Gottschalk, M. (2004). Extracellular virulence factors of streptococci associated with animal diseases. Front Biosci 9, $1157-1188$.

Segura, M. A., Cleroux, P. \& Gottschalk, M. (1998). Streptococcus suis and group B Streptococcus differ in their interactions with murine macrophages. FEMS Immunol Med Microbiol 21, 189-195.

Sharma, V., Gupta, R., Jhingran, A., Singh, B. P., Sridhara, S., Gaur, S. N. \& Arora, N. (2006). Cloning, recombinant expression and activity studies of a major allergen "enolase" from the fungus Curvularia lunata. J Clin Immunol 26, 360-369. 
Smith, H. E., Damman, M., van der Velde, J., Wagenaar, F., Wisselink, H. J., Stockhofe-Zurwieden, N. \& Smits, M. A. (1999). Identification and characterization of the cps locus of Streptococcus suis serotype 2: the capsule protects against phagocytosis and is an important virulence factor. Infect Immun 67, 1750-1756.

Talay, S. R., Zock, A., Rohde, M., Molinari, G., Oggioni, M., Pozzi, G., Guzman, C. A. \& Chhatwal, G. S. (2000). Co-operative binding of human fibronectin to $\mathrm{Sfbl}$ protein triggers streptococcal invasion into respiratory epithelial cells. Cell Microbiol 2, 521-535.

Tamura, G. S., Kuypers, J. M., Smith, S., Raff, H. \& Rubens, C. E. (1994). Adherence of group B streptococci to cultured epithelial cells: roles of environmental factors and bacterial surface components. Infect Immun 62, 2450-2458.

Tang, J., Wang, C., Feng, Y., Yang, W., Song, H., Chen, Z., Yu, H., Pan, X., Zhou, X. \& other authors (2006). Streptococcal toxic shock syndrome caused by Streptococcus suis serotype 2. PLoS Med 3, e151.

Teifel, M. \& Friedl, P. (1996). Establishment of the permanent microvascular endothelial cell line $\mathrm{PBMEC} / \mathrm{C} 1-2$ from porcine brains. Exp Cell Res 228, 50-57.

Tenenbaum, T., Adam, R., Eggelnpohler, I., Matalon, D., Seibt, A., Novotny, G. E., Galla, H. J. \& Schroten, H. (2005). Strain-dependent disruption of blood-cerebrospinal fluid barrier by Streptococcus suis in vitro. FEMS Immunol Med Microbiol 44, 25-34.

Tenenbaum, T., Essmann, F., Adam, R., Seibt, A., Janicke, R. U., Novotny, G. E., Galla, H. J. \& Schroten, H. (2006). Cell death, caspase activation, and HMGB1 release of porcine choroid plexus epithelial cells during Streptococcus suis infection in vitro. Brain Res 1100, $1-12$.
Valentin-Weigand, P., Talay, S. R., Timmis, K. N. \& Chhatwal, G. S. (1993). Identification of a fibronectin-binding protein as adhesin of Streptococcus pyogenes. Zentralbl Bakteriol 278, 238-245.

Vanier, G., Segura, M., Friedl, P., Lacouture, S. \& Gottschalk, M. (2004). Invasion of porcine brain microvascular endothelial cells by Streptococcus suis serotype 2. Infect Immun 72, 1441-1449.

Vanier, G., Segura, M. \& Gottschalk, M. (2007a). Characterization of the invasion of porcine endothelial cells by Streptococcus suis serotype 2. Can J Vet Res 71, 81-89.

Vanier, G., Sekizaki, T., Dominguez-Punaro, M. C., Esgleas, M., Osaki, M., Takamatsu, D., Segura, M. \& Gottschalk, M. (2007b). Disruption of srtA gene in Streptococcus suis results in decreased interactions with endothelial cells and extracellular matrix proteins. Vet Microbiol 127, 417-424.

Westerlund, B. \& Korhonen, T. K. (1993). Bacterial proteins binding to the mammalian extracellular matrix. Mol Microbiol 9, 687-694.

Wold, F. \& Ballou, C. E. (1957). Studies on the enzyme enolase. I. Equilibrium studies. J Biol Chem 227, 301-312.

Ye, C., Zhu, X., Jing, H., Du, H., Segura, M., Zheng, H., Kan, B., Wang, L., Bai, X. \& other authors (2006). Streptococcus suis sequence type 7 outbreak, Sichuan, China. Emerg Infect Dis 12, 1203-1208.

Yother, J. \& White, J. M. (1994). Novel surface attachment mechanism of the Streptococcus pneumoniae protein PspA. J Bacteriol 176, 2976-2985.

Zhang, W. \& Lu, C. P. (2007). Immunoproteomics of extracellular proteins of Chinese virulent strains of Streptococcus suis type 2. Proteomics 7, 4468-4476.

Edited by: T. J. Mitchell 